\title{
Direkte Demokratie in der Bundesrepublik Deutschland? - Ergebnisse einer Umfrage zur Einführung direkter Volksrechte auf Bundesebene
}

\author{
Lars P. Feld/Zohal Hessami/Lisa Reil
}

\section{A. Einleitung}

Das Urteil des Bundesverfassungsgerichts zum Vertrag von Lissabon vom 30. Juni 2009 löste in Deutschland ein geteiltes Echo unter Befürwortern und Gegnern des Vertrages aus. So begrüßte Paul Kirchhof in einem Gastbeitrag für die Frankfurter Allgemeine Zeitung das Urteil. Es setze „einen demokratischen Impuls für die Europäische Union“ und gebe ,dem Bürger mehr Rechtssicherheit gegenüber deutschem und europäischem Recht“". Hingegen kritisierten Christian Calliess und Martin Nettesheim in ihren Beiträgen in der gleichen Zeitung das Urteil scharf. $^{2}$ Das Bundesverfassungsgericht gehe auf Konfrontation mit dem Europäischen Gerichtshof (EuGH). Es sei „liebenswürdig, wie sich inzwischen einzelne Richter öffentlich dazu äußern, wie sie sich den Akt der Verfassungsneugebung durch Volksabstimmung ... im Einzelnen vorstellen. Aber der Widerspruch zwischen der Entmündigung der politisch handelnden legalen Staatsorgane und dem Vertrauen in die Vernunft revolutionärer Kraft ist auffällig." Christian Hillgruber sah sich daher bemüßigt, das Bundesverfassungsgericht ,gegen eine ins Maßlose gesteigerte, die Autorität des Gerichts in Frage stellende Kritik“ zu verteidigen. ${ }^{3}$ Das Gericht habe lediglich geltendes Verfassungsrecht angewendet.

Was rief diese harsche Kritik am Bundesverfassungsgericht hervor? Das Gericht hatte festgestellt, Verfassungsänderungen, die Kompetenzen an die Europäische Union abtreten und dabei den Bereich der Ewigkeitsgarantie tangieren, seien nicht ohne ein obligatorisches Referendum möglich, denn: „Ohne den ausdrücklich erklärten Willen der Völker sind die gewählten Organe nicht befugt, in

$1 \quad$ P. Kirchhof, Demokratie in Europa: Das neue Begleitgesetz zum Lissabon-Vertrag muss anspruchsvoll sein, in: FAZ Nr. 152 v. 4.7.2009, 12.

2 C. Calliess, Unter Karlsruher Totalaufsicht, in: FAZ Nr. 198 v. 27.8.2009, 8 und M. Nettesheim, Entmündigung der Politik, in: FAZ Nr. 198 v. 27.8.2009, 8 .

3 C. Hillgruber, Die besseren Europäer, in: FAZ Nr. 210 v. 10.9.2009, 8. 
ihren staatlichen Verfassungsräumen ein neues Legitimationssubjekt zu schaffen oder die vorhandenen zu delegitimieren. “4 Die verfassungsgebende Kompetenz im Hinblick auf die durch die Ewigkeitsgarantie gewährleisteten Grundrechte obliegt somit den Bundesbürgern selbst und nicht den sie repräsentierenden Organen: „Allein das souveräne deutsche Volk könnte dann durch einen revolutionären Akt kraft seiner ursprünglichen und unverlierbaren verfassunggebenden Gewalt seine eigene Grundentscheidung in einem verfassunggebenden Akt revidieren." ${ }^{\text {"5 }}$ Calliess kommentierte diesen direktdemokratischen Vorbehalt für die europäische Integration mit den Worten: „Wenn also die deutschen Bürger, repräsentiert durch eine verfassungsändernde Mehrheit von zwei Dritteln in Bundestag und Bundesrat, in Übereinstimmung mit dem Integrationsauftrag von Art. 23 Abs. 1 Satz 1 die EU fortentwickeln wollten, dann werden sie vom Bundesverfassungsgericht vor eine Wahl gestellt, die ein Großteil der Bürger nicht will: Sie müssten sich im Wege einer Volksabstimmung vom bewährten Grundgesetz zugunsten einer europäischen Oberverfassung verabschieden.“6 Das Bundesverfassungsgericht als Wegbereiter eines revolutionären Akts oder doch zumindest als populistischer Verhinderer der europäischen Integration?

Ein zweites Ereignis im Jahr 2009 hat massive grundsätzliche Kritik an der direkten Demokratie ausgelöst. Am 29. November 2009 nahmen 57,4 Prozent der Abstimmenden, bei einer relativ hohen Stimmbeteiligung von 53,4 Prozent und einer deutlichen Mehrheit der Kantone, die Verfassungsinitiative zum Verbot des Baus neuer Minarette (Minarettverbot) an. Mit der einfachen Mehrheit der Abstimmenden und dem Kantonsmehr ist diese Initiative nunmehr geltendes Verfassungsrecht in der Schweiz, sofern sie nicht vom Europäischen Gerichtshof für Menschenrechte gekippt wird. Nicht nur in der islamischen Welt löste diese Entscheidung einen Aufschrei aus. In mehreren europäischen Ländern, auch in Deutschland, wurde massive Kritik geübt. Der Economist stellte in seiner Berichterstattung klar, wie die Befürworter des Minarettverbots einzuordnen seien: „The result was not what was expected by decent, right-thinking people... ${ }^{67} \mathrm{Ge}-$ nerell müsse von einer ,awkward tradition of citizens' referendums ${ }^{6{ }^{68}}$ gesprochen

BVerfGE 123, 267 (404).

5 C. Calliess, Das Ringen des Zweiten Senats mit der Europäischen Union: Über das Ziel hinausgeschossen ..., in: ZEuS 12 (2009), 559 (575).

6 Calliess, Ringen (Fn. 5), S. 574.

7 The Return of the Nativists, in: The Economist Vol. 393 Nr. 8660 v. 5.12.2009, $61 \mathrm{f}$.

8 Charlemagne, The Swiss in the Middle, in: The Economist Vol. $393 \mathrm{Nr} .8660 \mathrm{v}$. 5.12.2009, 34. In einem Leserbrief kommentierte Bruno Frey diese Einlassung mit dem Hinweis, dass in anderen europäischen Ländern ähnliche Mehrheiten gegen den Bau von Moscheen bestehen würden, und schlug eine alternative Einschätzung des Schweizer 
werden. Günther Nonnenmacher, Herausgeber der Frankfurter Allgemeinen Zeitung, kommentierte: „Vielleicht bringt das die Anhänger von Volksabstimmungen in unserem Lande zum Nachdenken, die sich so gerne auf Mehrheitsmeinungen berufen, wenn es ihnen in den Kram passt, aber betreten schweigen, wenn ihnen die ,vox populi ‘ gegen den Strich geht. ... Die Schweizer müssen jetzt mit einem Ergebnis leben, das für ihr Land politischen und womöglich auch wirtschaftlichen Schaden stiftet. Darin muss ihnen Deutschland nicht nacheifern. "99 Andreas Ross stellte jedoch auch fest, dass es das gute Recht der Bürger sei, ihre Repräsentanten zu ermahnen, sie auch wirklich zu repräsentieren. ${ }^{10}$

Das sowohl in der Kritik am Lissabon-Urteil der Karlsruher Richter als auch am Schweizer Minarettverbot aufscheinende Unbehagen mit der direkten Demokratie aus juristischen und aus Medienkreisen findet sich in der wissenschaftlichen Debatte zu den Vor- und Nachteilen direkter Volksrechte wieder. Dort werden Argumente, die für und gegen direkte Demokratie im Vergleich zur reinen repräsentativen Demokratie sprechen, intensiv diskutiert. ${ }^{11}$ Die Analyse reicht von der Frage, ob Sachentscheidungen in Volksabstimmungen mit dem gleichen Informationsgehalt wie in der repräsentativen Demokratie getroffen werden, ${ }^{12}$ über die Rolle der Interessengruppen in beiden Demokratietypen ${ }^{13}$ und den potentiellen Konflikt mit völkerrechtlichen Verpflichtungen bis hin zur

Minarettverbots vor: ,the awkward tradition of parliamentary democracy in which politicians do not know, and do not want to know, the feelings and desires of their citizens". Siehe B.S. Frey, Letter on Popular Politics, in: The Economist Vol. 393 Nr. 8662 v. 19.12.2009, 20.

9 G. Nonnenmacher, Das Ende der Diskretion, in: FAZ Nr. 281 v. 3.12.2009, 1.

10 A. Ross, Volksaufbegehren, in: FAZ Nr. 291 v. 15.12.2009, 1.

11 Siehe dazu insbesondere H. Dreier $/ F$. Wittreck, Repräsentative und direkte Demokratie im Grundgesetz, in: L.P. Feld u.a. (Hrsg.), Jahrbuch für direkte Demokratie 2009, 2010, S. 11 ff. sowie den Band von H.K. Heußner/O. Jung (Hrsg.), Mehr direkte Demokratie wagen - Volksentscheid und Bürgerentscheid: Geschichte - Praxis - Vorschläge, 2. Aufl. 2009; aus ökonomischer Sicht G. Kirchgässner/L.P. Feld/M.R. Savioz, Die direkte Demokratie: modern, erfolgreich, entwicklungs- und exportfähig, 1999. Eine immer noch äußerst lesenswerte und ausgewogene Betrachtung aus Schweizer Rechtssicht findet sich in R.A. Rhinow, Grundprobleme der schweizerischen Demokratie, in: ZSR N.F. 103 (1984), $111 \mathrm{ff}$.

12 A. Lupia/M. McCubbins, The Democratic Dilemma, Cambridge 1998 sowie M. Benz/A. Stutzer, Are Voters Better Informed When They Have a Larger Say in Politics? Evidence for the European Union and Switzerland, in: Public Choice 119 (2004), $31 \mathrm{ff}$.

13 D.S. Broder, Democracy Derailed: Initiative Campaigns and the Power of Money, New York 2000. 
Problematik des Minderheitenschutzes ${ }^{14}$ und der ökonomischen Auswirkungen direkter Volksrechte (,Finanztabu der Volksgesetzgebung“).

In einem Beitrag zu direkter Demokratie und Menschenrechten stellt Kirchgässner klar, dass der grundlegende Konflikt zwischen Demokratieprinzip und Minderheitenschutz für jeden Demokratietypus gültig ist und kein besonderer Vor- oder Nachteil der repräsentativen gegenüber der direkten Demokratie besteht. ${ }^{15}$ Entscheidend ist letztlich, inwiefern Minderheitenrechte durch eine unabhängige Justiz, vor allem aber durch die Verfassungsgerichtsbarkeit geschützt werden. Dahingehend hat die Schweiz wohl ein Defizit, Deutschland hat es nicht. Die ökonomischen Analysen belegen hingegen relativ eindeutig die Vorteilhaftigkeit der direkten Demokratie und hier vor allem des Finanzreferendums. $^{16}$

14 T.E. Cronin, Direct Democracy: The Politics of Initiative, Referendum, and Recall, Cambridge 1989 und I. Budge, The New Challenge of Direct Democracy, Cambridge 1996.

15 G. Kirchgässner, Direkte Demokratie und Menschenrechte, in: Feld u.a., Jahrbuch 2009 (Fn. 11), S. 66 (73).

16 Siehe dazu im Überblick Kirchgässner/Feld/Savioz, Direkte Demokratie (Fn. 11); L.P. Feld/G. Kirchgässner, Direct Democracy, Political Culture, and the Outcome of Economic Policy: A Report on the Swiss Experience, in: EuJPolEcon 16 (2000), 287 ff.; dies., Fiscal Policy and Direct Democracy: Institutional Design Determines Outcomes, in: A.F. Ott/R.J. Cebula (Hrsg.), The Elgar Companion to Public Economics: Empirical Public Economics, Cheltenham 2006, S. 215 ff.; dies., Wirkungen direkter Demokratie Was sagt die politische Ökonomie?, in: Heußner/Jung, Direkte Demokratie (Fn. 11), S. 417 ff. Die frühen Klassiker dieser Literatur stammen von Werner Pommerehne: W.W. Pommerehne, Institutional Approaches to Public Expenditure: Empirical Evidence from Swiss Municipalities, in: Journal of Public Economics 9 (1978), 255 ff.; ders., Private versus öffentliche Müllabfuhr - nochmals betrachtet, in: Finanzarchiv 41 (1983), 466 ff. Seit den Neunziger Jahren findet sich eine Vielzahl von empirischen Analysen für die Schweiz und die USA. Siehe J.G. Matsusaka, Fiscal Effects of the Voter Initiative: Evidence from the Last 30 Years, in: Journal of Political Economy 103 (1995), 587 ff.; J.G. Matsusaka, For the Many or the Few: How the Initiative Process Changes American Government, Chicago 2004; ders., Direct Democracy and Public Employees, in: American Economic Review 99 (2009), 2227 ff. und E.R. Gerber, The Populist Paradox: Interest Group Influence and the Promise of Direct Legislation, Princeton 1999 für die USA, sowie L.P. Feld/M.R. Savioz, Direct Democracy Matters for Economic Performance: An Empirical Investigation, in: Kyklos 50 (1997), 507 ff.; L.P. Feld/G. Kirchgässner, Public Debt and Budgetary Procedures: Top Down or Bottom Up? Some Evidence from Swiss Municipalities, in: J. Poterba/J. v. Hagen (Hrsg.), Fiscal Institutions and Fiscal Performance, Chicago 1999, S. 151 ff.; dies., The Political Economy of Direct Legislation: Direct Democracy in Local and Regional Decision-Making, in: Economic Policy 33 (2001), 329 ff.; dies., Does Direct Democracy Reduce Public Debt? Evidence from Swiss Municipalities, in: Public Choice 109 (2001), 347 ff.; L.P. Feld/J.G. Matsusaka, Budget Referendums and Government Spending: Evidence from Swiss Cantons, in: Journal of Public Economics 87 (2003), 2703 ff.; P. Funk/C. Gathmann, Estimating the Effects of 
In diesem Beitrag geht es daher nicht darum, die längst bekannten Argumente zur Diskussion um direkte Demokratie im Lichte der jüngeren Debatten zu wiederholen. Vielmehr soll die Frage beleuchtet werden, ob und ggf. warum die Bürger mehr direkte Demokratie wollen. Calliess deutet an, dass die Bürger keine Verabschiedung vom Grundgesetz zugunsten einer europäischen Verfassung in einem Referendum wünschen, ohne jedoch dafür irgendwelche empirische Belege zu liefern. ${ }^{17}$ Uns interessiert nicht die enge verfassungs- und europapolitische Fragestellung, die das Grundgesetz gegen den Lissabonvertrag stellt, sondern, ob die Bürger eine Einführung direktdemokratischer Mechanismen auf der Bundesebene, die eine Voraussetzung für eine solche verfassungspolitische Entscheidung ist, generell wünschen. Der von Calliess hergestellte Gegensatz ist eine suggestive Überzeichnung des Kernproblems. Es geht in den weiteren Integrationsschritten auf europäischer Ebene nicht um die komplette Abschaffung des Grundgesetzes. Aber es ist notwendig, die Bundesbürger, gerade wenn es um den Kernbereich des Grundgesetzes geht, über Kompetenzverschiebungen auf die europäische Ebene mitentscheiden zu lassen. Eine schleichende Aushöhlung des Grundgesetzes und seine schrittweise Ablösung durch europäische Verträge sollten nicht ohne Genehmigung durch den Souverän erfolgen. Diese Sicht wird durch das Urteil der Karlsruher Richter zumindest implizit gestützt.

Direktdemokratische Mechanismen wie die Volksinitiative finden in Deutschland schließlich immer mehr Zuspruch. Obwohl manche westdeutsche Bundesländer direkte Volksrechte schon zum Teil seit 1946 (Bayern und Hessen) in ihren Verfassungen vorsehen, lässt sich der Trend zu mehr direkter Demokratie vor allem seit der Wiedervereinigung beobachten. Dieser Trend zeigt sich u.a. in der Einführung direkter Volksrechte in die Landesverfassungen, sofern sie noch nicht existierten, in der Erleichterung der Nutzung direktdemokratischer Mechanismen und in der Zunahme ihrer tatsächlichen Nutzung auf Landes- und Kommunalebene. ${ }^{18}$ Deutschland gilt jedoch bei der Umsetzung direktdemokratischer

Direct Democracy on Policy Outcomes: Preferences Matter!, in: mimeo, Stockholm Institute for Transition Economics 2005; dies., Does Direct Democracy Reduce the Size of Government? Evidence from Historical Data, 1890-2000, in: mimeo, Stanford University 2007; L.P. Feld/C.A. Schaltegger/J. Schnellenbach, On Government Centralization and Fiscal Referendums, in: European Economic Review 52 (2008), 611 ff.; C.A. Schaltegger/L.P. Feld, Do Large Cabinets Favor Large Governments?, Evidence from Sub-federal Jurisdictions. Journal of Public Economics 93 (2009), 35 ff.; L.P. Feld/J. Fischer/G. Kirchgässner, The Effect of Direct Democracy on Income Redistribution: Evidence for Switzerland, Economic Inquiry 48 (2010), 817 ff. für die Schweiz.

17 Calliess, Ringen (Fn. 5).

$18 \mathrm{Zu}$ den rechtlichen Rahmenbedingungen auf Landesebene und ihre Veränderung siehe vor allem die Dissertation von P. Neumann, Sachunmittelbare Demokratie im Bundes- 
Überzeugungen im internationalen Vergleich als zögerlich. Die Gründe für die Zustimmung zu oder die Ablehnung der direkten Demokratie in Deutschland wurden bislang noch nicht empirisch untersucht. Im Unterschied zu bereits bestehenden Umfragen geht es uns daher um die Frage, welche Faktoren die Zustimmung zu einer Einführung direktdemokratischer Elemente auf der Bundesebene beeinflussen. Um dies zu ergründen, betrachten wir zwei Akteursgruppen, die Bevölkerung und die Politiker, und orientieren uns an drei Kernfragen:

1. Wie steht die Bevölkerung zu einer Einführung direkter Demokratie auf Bundesebene, und was sind die Bestimmungsfaktoren dieser Einstellung?

2. Wie stehen die politischen Repräsentanten zu einer solchen Einführung, und was bestimmt ihre Haltung?

3. Lässt sich aus den Unterschieden oder Gemeinsamkeiten in den Einstellungen zur direkten Demokratie (und deren Bestimmungsfaktoren) zwischen den beiden Akteursgruppen auf die Wahrscheinlichkeit einer Einführung der direkten Demokratie schließen?

Um auf diese Fragen eingehen zu können, wird zunächst der Begriff der ,direkten“ Demokratie und entsprechender Elemente kurz erläutert. Zusätzlich wird in Abschnitt 2 basierend auf den Ergebnissen bestehender Umfragen der aktuelle Forschungsstand über die Einstellung der deutschen Bevölkerung zu direkten Volksrechten aufgezeigt. Zudem geben die Partei- und Grundsatzprogramme einen Überblick über die Positionen der Parteien zu diesem Sachverhalt. In Abschnitt 3 wird die Umfrage, die als Datengrundlage für diese Arbeit dient, vorgestellt. Durch die anschließenden deskriptiven Analysen in Abschnitt 4 wird ein erster Blick auf die Ergebnisse der Umfrage gewährt. In Abschnitt 5 werden die Ergebnisse einer multivariaten Regressionsanalyse berichtet, die Bestimmungsfaktoren der Einstellungen ermitteln sollen. Abschließend werden die drei Kernfragen beantwortet.

und Landesverfassungsrecht unter besonderer Berücksichtigung der neuen Länder, 2009. Kürzere Beiträge, die auch ein Licht auf die tatsächliche Nutzung werfen, liefern G. Jürgens $/ F$. Rehmet, Direkte Demokratie in den Bundesländern - Ein Überblick, in: Heußner/Jung, Direkte Demokratie (Fn. 11), S. 197 ff. sowie V. Mittendorf, Bürgerbegehren und Bürgerentscheide in Deutschland: Regelungen - Nutzungen - Analysen, ebda., S. $327 \mathrm{ff}$. 


\section{B. Die direkte Demokratie: Begriffsabgrenzung und Umfrageergebnisse}

I. Erläuterungen der direktdemokratischen Elemente

Direkte Demokratie ist ein schillernder Begriff, der ganz unterschiedlich verstanden wird. Manche Autoren bezeichnen damit die direkte demokratische Mitbestimmung der Bürger in Sachfragen über Volksinitiative und Referendum. Neumann spricht daher vorzugsweise von sachunmittelbarer Demokratie. ${ }^{19} \mathrm{Ge}-$ mäß Dreier und Wittreck zeichnet sich die direkte Demokratie hingegen dadurch aus, „daß Sach- oder Personalentscheidungen durch das Volk selbst gefällt werden, ohne daß die Entscheidungsfindung Repräsentanten überantwortet wird. “20 Die in Deutschland diskutierte Direktwahl des Bundespräsidenten würde demnach zur direkten Demokratie gehören. Genau wie bei Neumann soll direkte Demokratie in diesem Beitrag nur als direkte Sachentscheidung verstanden werden.

Solche Sachentscheidungen können in fakultativen oder in obligatorischen Verfahren erfolgen. Zu den fakultativen Volksentscheiden zählen die Volksinitiative $^{21}$ und das fakultative Referendum. Die Volksinitiative räumt die Möglichkeit ein, einen unter den Bürgern eigenständig erarbeiteten Gesetzentwurf oder eine Verfassungsänderung vorzulegen und darüber verbindlich abzustimmen. In der Regel ist die Volksinitiative mit einem Unterschriftenquorum verbunden, d.h. sie kommt nur zur Abstimmung, wenn eine Mindestzahl an Unterschriften für ihr Zustandekommen geleistet wird. Angesprochen werden dabei oftmals Themen, die ansonsten im Parlament keine Beachtung finden, im Hinblick auf die jedoch von der Bevölkerung ein dringender Reformbedarf gesehen wird. Das fakultative Referendum ermöglicht den Bürgern, Entscheidungen des Gesetzgebers zu revidieren, bevor sie verbindliches Recht werden. Auch für sein Zustandekommen gilt ein Unterschriftenquorum. Das Referendum stellt ein Vetorecht der Bevölkerung dar, dessen Bedeutung insbesondere in seiner antizipativen und disziplinierenden Wirkung des Parlamentes liegt.

Als obligatorischer Volksentscheid ist das obligatorische Referendum zu nennen, welches ein verfassungsrechtlich ausgelöstes Verfahren in Fragen darstellt, bei denen auf die Zustimmung des Volkes nicht verzichtet werden kann oder soll. Auf Bundesebene ist dies lediglich bei der Neugliederung des Staatsgebietes

19 Neumann, Sachunmittelbare Demokratie (Fn. 18).

20 Dreier/Wittreck, Repräsentative und direkte Demokratie (Fn. 11), S. 18.

21 Synonym wird der Begriff Volksinitiative auch mit dem Begriff Volksbegehren oder im Dreiklang, Volksinitiative, Volksbegehren und Volksentscheid verwendet. 
nach Art. 29 GG der Fall. Das obligatorische Referendum kann sich sowohl auf Gesetze als auch auf Total- oder Teilrevisionen der Verfassung beziehen. Entscheidend ist, dass vorab bestimmt ist, in welchen Fragen Volksabstimmungen stattfinden müssen, und dass diese bindend sind. Ein Unterschriftenquorum existiert bei obligatorischen Volksentscheiden folgerichtig nicht.

$\mathrm{Zu}$ den konsultativen Volksentscheiden zählen die konsultative Volksinitiative und das konsultative Referendum. Es handelt sich dabei um eine von der Bevölkerung beantragte und formulierte Initiative bzw. ein von der Bevölkerung beantragtes Referendum, die beide jedoch keine Verbindlichkeit, sondern nur beratenden Charakter haben. Ein verbindliches Einwirken auf die Gesetzgebung wird somit nicht erreicht. Gleiches gilt für die durch die Staatsorgane ausgelöste Volksbefragung. Ein weiteres Element ist das Plebiszit, das ebenfalls oft unter der Kategorie der fakultativen Volksentscheide angeführt wird. Es sollte jedoch nicht zu den Elementen der direkten Demokratie gezählt werden, da es durch Organe der repräsentativen Demokratie (Präsident, Regierung, Parlament) initiiert wird.

Diese grundlegenden Elemente der direkten Demokratie können unterschiedlich ausgestaltet sein. ${ }^{22}$ So werden bestimmte Themen, wie etwa Finanzfragen, nicht selten generell der direkten Demokratie entzogen. Auch können Unterschriftenquoren unterschiedlich hoch sein. In der Schweiz liegen sie für die Verfassungsinitiative bei ca. 2\% auf der Bundesebene und variieren von Kanton zu Kanton. In Deutschland findet sich für das Volksbegehren eine Spanne von $4 \%$ in Brandenburg bis $20 \%$ in Hessen und im Saarland. Zehn von 16 Bundesländern liegen mit ihrem Unterschriftenquorum bei mindestens 10\%. Auf Landesebene beläuft sich die Zeitspanne zur Sammlung der Unterschriften auf 14 Tage in Hessen, während Mecklenburg-Vorpommern keine Frist hat. In der Schweiz können 18 Monate lang Stimmen gesammelt werden. Zudem bestehen in Deutschland neben dem Unterschriftenquorum auch Zustimmungsquoren, wonach ein bestimmter Prozentsatz der Stimmberechtigten für den Gesetzentwurf stimmen muss. Bei einfachen Gesetzen liegen sie zwischen 15 Prozent in Nordrhein-Westfalen und 50 Prozent im Saarland. Bayern, Hessen und Sachsen sehen kein Zustimmungsquorum vor.

22 Siehe zu den folgenden Angaben Jürgens/Rehmet, Direkte Demokratie in den Bundesländern (Fn. 18). 
Die bislang in Deutschland durchgeführten Befragungen zur Einführung der direkten Demokratie auf Bundesebene konzentrieren sich auf die Bevölkerung. Gemäß dreier repräsentativer Umfragen des Instituts für Demoskopie Allensbach sprachen sich 1998, 2000 und 2010 mehr als die Hälfte der Deutschen für Volksabstimmungen auf Bundesebene aus (siehe Tabelle 1). Diese Zustimmung stieg zwischen 1998 und 2000 leicht von 51 Prozent auf 55 Prozent, um wieder auf 51 Prozent im Jahr 2010 zurückzugehen. Der Anteil derjenigen, die direkte Demokratie auf Bundesebene ablehnen, nahm im gleichen Zeitraum um drei Prozentpunkte $a b$. Unter den Bürgern in den neuen Bundesländern ist mit mehr als zwei Dritteln der Befragten der Wunsch nach direkter Demokratie deutlich stärker als in Westdeutschland ausgeprägt. In der Nachbefragung des Allensbacher Institutes $^{23}$ gaben 71 Prozent der Befragten zudem an, dass Volksabstimmungen einen guten Einfluss auf die Demokratie in Deutschland hätten. In der jüngsten Befragung von 2010 zeigen sich 65 Prozent der Befragten überzeugt, dass die Qualität der direkten Demokratie durch die Einführung direkter Volksrechte verbessert würde. ${ }^{24} \mathrm{Zu}$ ähnlichen Ergebnissen kommen andere Umfragen, etwa von TNSEmnid (2002). Demnach befürworten sogar knapp 83 Prozent die Einführung von Volksentscheiden auf Bundesebene.

Tabelle 1: Präferenzen zur repräsentativen oder zur direkten Demokratie

\begin{tabular}{l|rrrrrrrr}
\hline & \multicolumn{3}{|c}{$\mathbf{1 9 9 8}$} & & $\mathbf{2 0 0 0}$ & 2010 \\
\hline & West & Ost & Gesamt & West & Ost & Gesamt & Gesamt \\
Repräsentative Demokratie & 39 & 18 & 35 & 37 & 17 & 33 & 32 \\
Direkte Demokratie & 47 & 67 & 51 & 52 & 68 & 55 & 51 \\
\hline Nicht sicher & 14 & 15 & 14 & 11 & 15 & 12 & 17 \\
\hline
\end{tabular}

Quellen: E. Noelle-Neumann, Was ist anders als 1994? Deutschland verwandelt sich. Dokumentation des Beitrages in der FAZ Nr. 47 v. 25.2.1998, Institut für Demoskopie Allensbach. E. Noelle-Neumann/R. Köcher, Allensbacher Jahrbuch der Demoskopie 19982002, Bd. 11, 2002, R. Köcher, Der Ruf nach dem Plebiszit, in: FAZ v. 20.10.2010, 5, ba-

23 E. Noelle-Neumann/R. Köcher, Allensbacher Jahrbuch der Demoskopie 1998-2002, Bd. 11, 2002.

24 R. Köcher, Der Ruf nach dem Plebiszit, in: FAZ v. 20.10.2010, 5. 
sierend auf Daten des Allensbacher Institutes: Anteil der Befürworter des jeweiligen Demokratiemodells in Prozent.

Politische Entscheidungsträger wurden bislang nicht zu ihren Einstellungen zur Einführung direkter Demokratie auf Bundesebene befragt. Allerdings zeigt eine Analyse auf Basis der europäischen Wahlstudie 2009, die auf einer Befragung der Bevölkerung und der Kandidaten bei den Wahlen zum Europaparlament in den 27 Mitgliedstaaten der EU basiert, eine deutlich höhere Zustimmung zu Referenden auf Seiten der Bürger als auf Seiten der Kandidaten. ${ }^{25}$ Im EUDurchschnitt liegt die Zustimmungsrate der Bürger um mehr als 30 Prozentpunkte höher als die der Kandidaten. In Deutschland beträgt diese Differenz 46 Prozentpunkte, in Irland sogar 65 Prozentpunkte. Interessanterweise sind die $\mathrm{Zu}$ stimmungsraten zu Referenden bei Entscheidungen über EU-Verträge von Befürwortern und Gegnern der europäischen Integration nicht sehr unterschiedlich. 77,3 Prozent der Bürger, die sich dagegen aussprechen, die europäische Einigung voranzutreiben, sprechen sich für EU-Referenden aus. Bei den Befürwortern einer weiteren Integration liegt die Zustimmungsrate bei 68,6 Prozent.

Zudem liefern die Parteiprogramme der im Deutschen Bundestag vertretenen Parteien sowie das Abstimmungsverhalten der Abgeordneten Anhaltspunkte für die Positionen der politischen Parteien, zuletzt bei einer Ablehnung der Gesetzentwürfe von FDP, Bündnis 90/Die Grünen und der Linken zur Aufnahme von Volksinitiative, Volksbegehren und Volksentscheid in das Grundgesetz am 23. April 2009. So treten gemäß den Grundsatzprogrammen der Parteien außer der CDU/CSU alle anderen im Bundestag vertretenen Parteien für direkte Demokratie auf Bundesebene ein. Hinsichtlich der Details der Umsetzung unterscheiden sich die Befürworter jedoch. So schlägt die FDP in ihrem in der vergangenen Legislaturperiode vorgelegten Gesetzentwurf ein Unterschriftenquorum von 400.000 Stimmberechtigten für die Volksinitiative sowie zehn Prozent der Wahlberechtigten für das Zustandekommen des Volksbegehrens und ein $\mathrm{Zu}$ stimmungsquorum für Gesetze von 15 Prozent sowie für Grundgesetzänderungen von 25 Prozent der Wahlberechtigten vor. Volksinitiativen über Haushaltsoder Abgabengesetze sind unzulässig. Allerdings sind finanzwirksame Volksinitiativen möglich, wenn sie mit einem kostendeckenden Finanzierungsplan versehen sind. Der Gesetzentwurf der Grünen stimmt mit diesem Vorschlag im Wesentlichen überein, fordert jedoch keinen Finanzierungsplan und legt eine Hürde von fünf Prozent der Wahlberechtigten für das Zustandekommen des Volksbegehrens fest. Der Gesetzentwurf der Linken enthält ein Unterschriftenquorum

25 Siehe dazu H. Giebler, Starke Nachfrage: Bürger wollen mehr EU-Referenden - die meisten Politiker zögern, in: WZB Mitteilungen 130 (2010), 14. 
von 100.000 Wahlberechtigten für die Volksinitiative, einer Million für das $\mathrm{Zu}-$ standekommen eines Volksbegehrens über Gesetze und zwei Millionen für Grundgesetzänderungen. Ein Zustimmungsquorum für Gesetze findet sich nicht, aber ein Beteiligungsquorum von 25 Prozent für Grundgesetzänderungen.

Trotz dieser Unterschiede belegt das Abstimmungsverhalten über diese Gesetzentwürfe ein ernsthaftes Eintreten der FDP, der Grünen und der Linken für direkte Demokratie auf Bundesebene. Die SPD gab in der Bundestagsdebatte zu Protokoll, dass sie für direkte Demokratie auf Bundesebene sei. In der Abstimmung zeigte sich jedoch, dass Teile der SPD zusammen mit der CDU/CSU gegen die Gesetzentwürfe stimmten. Die CDU/CSU lehnt direkte Demokratie auf Bundesebene $a b$.

\section{Befragungsdesign und Methode}

In der vorliegenden Untersuchung sollen die Einstellungen der Politiker und der Bürger zur direkten Demokratie auf Bundesebene näher beleuchtet werden. Durch eine Differenzierung zwischen beiden Akteursgruppen können aussagekräftigere Ergebnisse erreicht werden, da unterschiedliche Tendenzen zur direkten Demokratie beider Gruppen zu vermuten sind. Ein breites Fragenportfolio zur direkten Demokratie erlaubt es zudem, näher auf Befürchtungen im Hinblick auf direkte Demokratie, den Reformwillen, das Wissen und das Blockadepotential der Politiker einzugehen. Somit ist es möglich, die Einstellungen zur direkten Demokratie sowie dazugehörige Einflussfaktoren zu identifizieren.

Zunächst wird durch deskriptive Analysen ein Überblick über die Befragung gegeben, um dann in einer Regressionsanalyse darauf einzugehen, welche Faktoren die Einstellung zur direkten Demokratie signifikant beeinflussen. Um Einstellungen der Bevölkerung und der Politiker zur direkten Demokratie und deren Motive zu analysieren, wurde folgender Ansatz gewählt. Die für die Bevölkerung zu prüfenden Einflusskategorien umfassen individuelle Charakteristiken, das Reformpotential, Politikverdrossenheit sowie die Ängste der Bevölkerung die direkte Demokratie betreffend. Durch zusätzliche oder leicht differierende Fragestellungen an die Politiker werden zu diesen Einflusskategorien der Bevölkerung noch ideologische Präferenzen und der Einfluss des Blockadepotentials einbezogen. 


\begin{tabular}{l|l}
\hline Fragestellungen & Beobachtungen \\
\hline & Politiker Bürger \\
Abhängige Variable &
\end{tabular}

Wie bewerten Sie im Großen und Ganzen eine Einführung der direkten Demokratie in Deutschland auf Bundesebene?

Soziodemographische bzw. politische Kontrollvariablen

Geschlecht

Alter

280

Höchster Bildungsabschluss

Wohnsitz

Politiker auf Kommunal-/Landes- oder Bundesebene

Parteizugehörigkeit

Variablen zum Reform-/Blockadepotential

Sind Sie mit den jetzigen Möglichkeiten zur politischen Mitbestimmung zufrieden?

Verbinden Sie mehr Mitbestimmung mit direkter Demokratie auf Bundesebene?

Fühlen Sie sich bei einer Einführung der direkten Demokratie auf Bundesebene in Deutschland in Ihrer Souveränität eingeschränkt?

Bürger sind bei direktdemokratischen Entscheidungen auf Bun
desebene zu leicht von Interessengruppen zu manipulieren.

Die größten Probleme bei der Einführung der direkten Demokratie in Deutschland auf Bundesebene wird der fehlende Wille der Bürger auf Grund von höheren Informationskosten und Verantwortung sein.

Auf Bundesebene muss gerade von den Politikern die Neuerung zur direkten Demokratie ausgehen, deren Macht und Einfluss

\section{Variablen zur Politikverdrossenheit}

Wenn meine Mitspracherechte durch die Einführung der direkten Demokratie auf Bundesebene erweitert werden, würde ich mehr hinter der Politik Deutschlands stehen. 
Als abhängige Variable verwenden wir in der Regressionsanalyse die persönliche Einstellung beider Gruppen zu einer Einführung der direkten Demokratie auf Bundesebene. Diese Frage steht am Ende des Fragebogens, so dass ein gewisser Lern- und Erkenntnisprozess durch die Beschäftigung mit dem Thema direkte Demokratie erfolgen kann. Daher wird der Begriff „Einstellung zur direkten Demokratie“ synonym für die abhängige Variable verwendet. In Tabelle 2 sind die Fragen aufgelistet, welche die für die Regression verwendeten erklärenden Variablen liefern. Zusätzlich wird die Anzahl der Beobachtungen angegeben. $^{26}$

Neben den sozio-demographischen Kontrollvariablen interessieren uns als erklärende Variablen des Regressionsmodells auf Seiten der Bürger der Wunsch nach direkter politischer Mitbestimmung und das Ausmaß an Politikverdrossenheit. Russell et al. argumentieren, durch die ansteigende Unzufriedenheit sowie das Misstrauen in die Institutionen der repräsentativen Demokratie werde der Wunsch nach direkter Demokratie verstärkt. ${ }^{27}$ Demnach dürfte eine hohe Zufriedenheit mit dem Ausmaß an politischer Mitbestimmung in der repräsentativen Demokratie die Zustimmung zu direkter Demokratie auf Bundesebene auf Seiten der Bürger senken, während eine hohe Politikverdrossenheit zu höherer Zustimmung führt.

Hinsichtlich der Ängste und Befürchtungen von Politikern greifen wir zwei Argumente gegen direkte Demokratie auf: Wird ein stärkerer Interessengruppeneinfluss in der direkten Demokratie erwartet, so dürfte die Zustimmung zu ihrer Einführung auf Bundesebene sinken. Sehen die Befragten ein Problem in den hohen Informationskosten der direkten Demokratie, so dürften sie sich ebenfalls eher gegen direkte Demokratie aussprechen. ${ }^{28}$ Auf Seiten der Politiker dürfte zudem der befürchtete Souveränitätsverlust bei Einführung direktdemokratischer Mechanismen den Widerstand gegen diese bedingen. ${ }^{29}$ Angesichts der Einstellungen der Parteien im vorangegangen Abschnitt überprüfen wir auch die Hypothese, dass Mitglieder konservativer Parteien die Einführung direkter Demokratie auf Bundesebene ablehnen.

Da die abhängige Variable eine binäre Variable ist, verwenden wir ein ProbitModell. Die geschätzten Koeffizienten und Standardfehler müssen hier, anders

26 Der gesamte Fragebogen ist auf Anfrage von den Autoren erhältlich.

27 D.J. Russell/W. Bürklin/A. Drummond, Public Opinion and Direct Democracy, in: Journal of Democracy 12 (2001), $141 \mathrm{ff}$.

28 Kirchgässner/Feld/Savioz, Direkte Demokratie (Fn. 11).

29 R. Ingelhart/C. Welzel, Modernization, Cultural Change, and Democracy: The Human Development Sequence, Cambridge 2005. 
als bei der linearen Regression in marginale Effekte umgerechnet werden, um sie quantitativ interpretieren zu können. Der Existenz heteroskedastischer Standardfehler wird durch die Verwendung robuster Standardfehler Rechnung getragen. Da die Stichprobe teilweise nicht proportional zum Verhältnis der Bevölkerungsgröße der Bundesländer oder der Parteigrößen ist, wird ausschließlich für West- und Ostdeutschland sowie linke und rechte Parteien kontrolliert.

\section{Deskriptive Evidenz}

\section{Durchführung der Befragung}

Die Befragung wurde im Jahr 2008 durchgeführt. Befragt wurden die bundesdeutsche Bevölkerung sowie Politiker aller politischen Ebenen mit separaten, ähnlich aufgebauten Fragebögen. Die Rücklaufquote der Politikerbefragung belief sich mit 280 Teilnehmern auf 23,2\%. Bei der telefonischen Befragung der Bevölkerung konnten lediglich 173 Fragebögen in der Analyse verwendet werden. Die zu befragenden Bürger wurden durch eine Wahrscheinlichkeitsauswahl bestimmt, bei der alle Einwohner eines Bundeslandes mit einem Festnetzanschluss die gleiche Chance haben, ausgewählt zu werden. Durch einen Schwerpunkt der Befragung nach 17.00 Uhr wurde sichergestellt, dass der Beruf und die Beschäftigungssituation der Befragten die Auswahlwahrscheinlichkeit nicht beeinflusst. Im Gegensatz zur Bevölkerungsbefragung fand die Befragung der Politiker postalisch statt. Es wurden Politiker der Kommunal-, Landes- und Bundesebene mit Hilfe eines Zufallsmechanismus ausgewählt, durch den alle die gleiche Chance hatten, unter den amtierenden Politikern im jeweiligen Bundesland beziehungsweise im Bundestag ausgewählt zu werden. Es wurden jeweils 400 Politiker auf jeder Ebene angeschrieben (insgesamt 1200). Die Gesamtzahl der Befragten verteilt sich wie folgt auf die politischen Parteien und Regierungsebenen:

Tabelle 3: Verteilung der befragten Politiker auf Parteien und politische Ebenen

\begin{tabular}{l|clclc}
\hline \multicolumn{1}{c|}{ Partei } & $\begin{array}{c}\text { Beobach- } \\
\text { tungen }\end{array}$ & Partei & $\begin{array}{c}\text { Beobach- } \\
\text { tungen }\end{array}$ & Politische Ebene & $\begin{array}{c}\text { Beobach- } \\
\text { tungen }\end{array}$ \\
\hline CDU & 63 & LINKE & 14 & Kommunalebene & 105 \\
CSU & 30 & SPD & 93 & Landesebene & 115 \\
FDP & 20 & Parteilos & 23 & Bundesebene & 58 \\
\hline GRÜNE & 26 & Sonstige & 5 & & \\
\hline
\end{tabular}


Anmerkung: Gesamtzahl weicht von den 280 Befragten ab, da Fragen teilweise nicht beantwortet wurden.

Da Bürger und Politiker unterschiedlich von direktdemokratischen Reformen betroffen wären, wurden zwei unterschiedliche Fragebögen entwickelt, die gruppenspezifische Befragungsschwerpunkte setzen. Alle Fragen waren mit geringer Abstufung zu beantworten (z.B.: Ja/Nein oder Stimme eher zu/Stimme eher nicht zu). Um die Verlässlichkeit der Befragungen zu testen, wurde ein in der Psychologie anerkanntes Maß der Reliabilität angewandt. ${ }^{30}$ Es handelt sich bei der Reliabilität um eines der drei Gütekriterien für empirische Untersuchungen. Hierzu wurde ein Korrelationskoeffizient zweier ,,paralleler“ Fragen gebildet. ${ }^{31}$ Während das Ergebnis der Politiker mit einer Reliabilität von 0,87 als sehr gut bezeichnet werden kann, ist bei der Bevölkerung ein geringerer Wert von 0,67 festzustellen. Da jedoch besonders bei den Bürgern, die nicht sehr vertraut mit dem Thema der direkten Demokratie sind, ein starker Lerneffekt während der Befragung zu vermuten ist, kann insgesamt eine niedrigere Reliabilität als bei den Politikern erwartet werden. Insofern dürfte der Wert von 0,67 nicht auf Messfehler und eine damit verbundene mangelnde Konsistenz hinweisen, sondern eher eine angemessene Höhe haben. Zusätzlich werden ausschließlich robuste Antworten in die Analyse aufgenommen, die nicht auf ein falsches Verständnis der Frage oder mehrmaliges Nachfragen während der Befragung oder andere UnregelmäBigkeiten hinweisen.

\section{Deskriptive Befragungsergebnisse}

Wie Tabelle 4 zeigt, lässt sich eine starke Zustimmung zur direkten Demokratie auf Bundesebene bei der Bevölkerung (75\%) und eine Abneigung bei den Politikern (44\%) feststellen. Ersteres steht im Einklang mit den Befragungsergebnissen des Allensbacher Institutes zur direkten Demokratie, wenn auch der Zustimmungsanteil in dieser Untersuchung höher ausfällt. Die Aufteilung Deutschlands in Ost und West, Süd und Mittel/Nord, kleine und große wie auch direktdemokratisch erfahrene und unerfahrene Länder ergibt weitere interessante Ergebnisse. So ist die positive Einstellung zu direktdemokratischen Mechanismen in Ost-

30 Siehe H.T. Kranz, Einführung in die klassische Testtheorie, 1981.

31 Zur Definition der Parallelität zweier Fragen siehe bereits die Übersicht von G. Fischer, Einführung in die Theorie psychologischer Tests, 1974. 
deutschland stärker ausgeprägt als in Westdeutschland. ${ }^{32}$ Dies gilt in stärkerem Maße für Politiker als für Bürger. Die Unterteilung in Süd- und Mittel-/Norddeutschland wurde vorgenommen, um einen möglichen Einfluss der geographischen Nähe zur Schweiz zu berücksichtigen. Dabei scheint ein negativer Einfluss des Vorbilds der Schweiz auf die Nachbarländer erkennbar. Jedoch könnte dies auch durch die in Bayern und Baden-Württemberg bestehende konservative Regierung zustande kommen, denn in Ländern mit konservativen Regierungen sprechen sich die Bevölkerung und die Politiker in geringerem Maße für die Einführung der direkten Demokratie auf Bundesebene aus.

Tabelle 4: Persönliche Einstellung gegenüber der Einführung der direkten Demokratie auf Bundesebene

\begin{tabular}{|c|c|c|c|c|}
\hline & \multicolumn{2}{|c|}{ Bevölkerung } & \multicolumn{2}{|c|}{ Politiker } \\
\hline & Mittelwert & Beobachtungen & Mittelwert & Beobachtungen \\
\hline Gesamtdeutschland & 0,75 & 152 & 0,44 & 271 \\
\hline Westdeutschland & 0,74 & 114 & 0,42 & 233 \\
\hline Ostdeutschland & 0,79 & 38 & 0,70 & 30 \\
\hline Süddeutschland & 0,60 & 20 & 0,35 & 94 \\
\hline Mittel-/Norddeutschland & 0,77 & 132 & 0,50 & 169 \\
\hline Konservativ regierte Länder & 0,68 & 92 & 0,42 & 204 \\
\hline Linksregierte Länder & 0,85 & 60 & 0,54 & 59 \\
\hline Kleine Länder & 0,83 & 69 & 0,59 & 70 \\
\hline Große Länder & 0,69 & 83 & 0,40 & 193 \\
\hline $\begin{array}{l}\text { direktdemokratisch } \\
\text { erfahrene Länder }\end{array}$ & 0,80 & 35 & 0,42 & 76 \\
\hline $\begin{array}{l}\text { direktdemokratisch } \\
\text { unerfahrene Länder }\end{array}$ & 0,74 & 117 & 0,46 & 187 \\
\hline
\end{tabular}

Quelle: Eigene Berechnung. Mittelwerte der binären Variablen können als prozentuale positive Einstellung interpretiert werden.

32 Siehe dazu auch W. Bürklin/H. Rebensdorf, Eliten in Deutschland: Rekrutierung und Integration, 1997, S. 391 ff., die dies auf einen stärkeren Einfluss der neuen Protestgeneration unter den Eliten Ostdeutschlands zurückführen. 
Dass sich sowohl die Bevölkerung als auch die Politiker der kleinen Bundesländer direktdemokratische Mechanismen eher vorstellen können, ist plausibel. So bewerten Einwohner großer Bundesländer die Schwierigkeiten einer effizienten Volksbeteiligung deutschlandweit anders als Einwohner eines kleinen Bundeslandes. Interessant ist, dass Bürger, die mit direkter Demokratie auf Landesebene vertraut sind, deren Einführung auf Bundesebene eher befürworten. Politiker, die bereits Erfahrung mit direktdemokratischen Mechanismen auf Landesebene haben, stehen der direkten Demokratie auf Bundesebene hingegen kritischer gegenüber. Überdies vermuten die befragten Politiker, dass durchschnittlich 50 Prozent der Bevölkerung für die Einführung der direkten Demokratie auf Bundesebene sind. Die Einschätzung, inwiefern direkte Demokratie tatsächlich eingeführt werden kann, fällt sowohl bei der Bevölkerung als auch bei den Politikern erheblich geringer aus.

Abbildung 1: Halten Sie es für sinnvoll, die direkte Demokratie in Deutschland auf Bundesebene einzuführen?

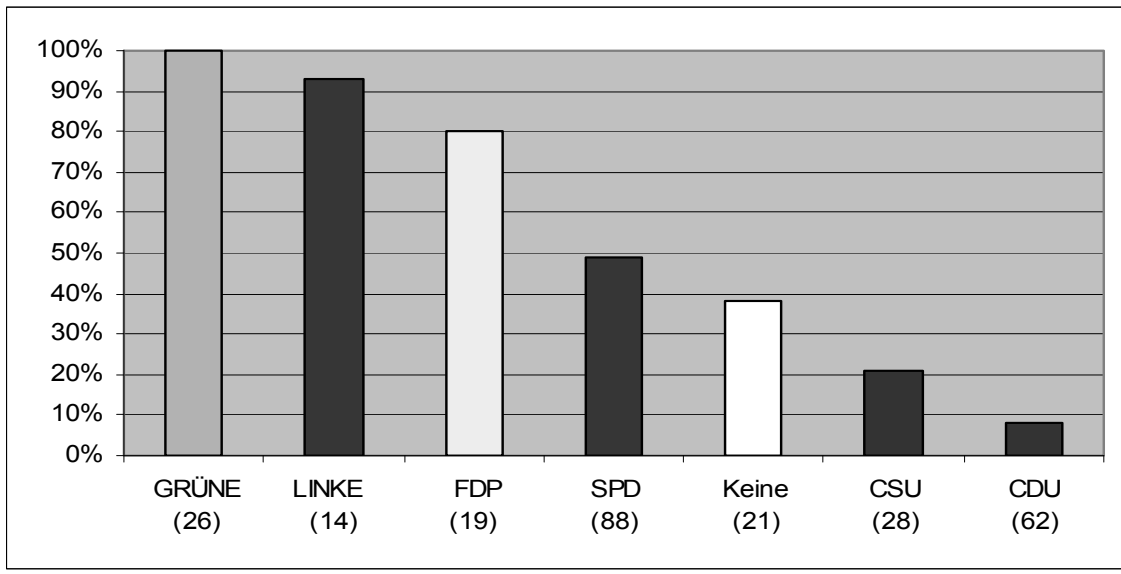

Quelle: Eigene Berechnung; Anteil der parteizugehörigen Politiker, die zustimmen. In Klammern: Anzahl der Politiker, die befragt wurden.

Abbildung 1 verdeutlicht, dass die Einstellung zur direkten Demokratie ideologisch geprägt ist. Der direkten Demokratie stehen CDU (dafür: 8,2\%) und CSU (dafür: 18,5\%) am stärksten kritisch gegenüber. Die größten Befürworter findet man unter den Grünen (dafür: 100\%) und den LINKEN (dafür: 92,3\%) gefolgt von der FDP mit 80\%. Die SPD zeigt mit 50\% ein gemischtes Bild. Auffällig ist, dass $32 \%$ der Grünen eine Einführung der direkten Demokratie für realistisch 
halten. Alle anderen Parteien stimmen dieser Einschätzung mit weit weniger als $32 \%$ zu. Da direkte Demokratie eines der drei Gründungsprinzipien der Grünen darstellt, ist eine solche positive Einstellung zu erwarten.

Tabelle 5: Der Wille zur politischen Beteiligung

\begin{tabular}{l|l}
\hline 1. Die Bürger wollen sich mehr informieren, sagen: & $\mathbf{5 7 \%}$ \\
\hline 2. & Bei direkten Mitspracherechten besteht eher die Bereitschaft, sich mit politischen \\
Themen auseinanderzusetzen, sagen: & $\mathbf{7 1 \%}$ \\
3. $\begin{array}{l}\text { Entscheidungen, die die Bürger direkt mitbestimmt haben, werden eher akzeptiert, } \\
\text { sagen: }\end{array}$ & $\mathbf{8 2 \%}$ \\
4. $\begin{array}{l}\text { Durch die Erweiterung meiner Mitspracherechte bei Einführung der direkten Demo- } \\
\text { kratie auf Bundesebene ... }\end{array}$ & $\mathbf{5 3 \%}$ \\
a) $\ldots$ werde ich mich regelmäßiger an Wahlen beteiligen, sagen: & $\mathbf{6 5 \%}$ \\
b) $\ldots$ werde ich mich intensiver über die politische Lage informieren, sagen: & $\mathbf{6 2 \%}$ \\
c) $\ldots$ bin ich den politischen Entscheidungen der Repräsentanten nicht mehr ausgeliefert, \\
sagen: & $\mathbf{7 7 \%}$ \\
d) $\ldots$ würde ich politische Entscheidungen eher akzeptieren, sagen: & $\mathbf{6 8 \%}$ \\
\hline e) $\ldots$ würde ich mehr hinter der Politik Deutschlands stehen, sagen: &
\end{tabular}

Quelle: Eigene Darstellung. Anteil der zustimmenden Bevölkerung.

Ein wichtiger Grund für die Zustimmung zur direkten Demokratie könnte die Politikverdrossenheit der Bürger sein. Gemäß den Befragungsergebnissen dürften mehr politische Mitbestimmungsmöglichkeiten eine Reduzierung der Politikverdrossenheit bedingen (Tabelle 5). So ist zu erkennen, dass durch die Möglichkeit, Einfluss auf die Politik zu nehmen und somit den Entscheidungen der Politiker nicht mehr ausgesetzt zu sein (Frage 4c), das Interesse an der Politik (2), die Informationsbereitschaft $(1,4 \mathrm{~b})$ und auch die Aktionsbereitschaft (4a) der Bevölkerung wieder deutlich steigen würden. Frage 4e zeigt zudem, dass durch die persönliche Beteiligung an Entscheidungen eine stärkere Identifizierung mit dem Staat erfolgt. 
Tabelle 6: Befürchtungen der Politiker und der Bevölkerung

\begin{tabular}{|c|c|c|}
\hline Befürchtungen & Politiker & Bevölkerung \\
\hline $\begin{array}{l}\text { Volksentscheide verengen Entscheidungen auf ein } \\
\text { schlichtes ,Ja“ oder „Nein“. }\end{array}$ & 81 & 53 \\
\hline $\begin{array}{l}\text { Auf Bundesebene muss von den Politikern die Neuerung } \\
\text { zur direkten Demokratie ausgehen, deren Macht dadurch } \\
\text { beschnitten wird. }\end{array}$ & 60 & 71 \\
\hline $\begin{array}{l}\text { Die Durchsetzung der Gesetzesvorlage zur dir. Dem. } \\
\text { durch die Politiker im Bundestag und Bundesrat stellt ein } \\
\text { Problem dar. }\end{array}$ & 75 & 85 \\
\hline $\begin{array}{l}\text { Die ansteigende Macht der Interessengruppen bei } \\
\text { Einführung der direkten Demokratie ist problematisch. }\end{array}$ & 81 & 75 \\
\hline $\begin{array}{l}\text { Bei direktdemokratischen Entscheidungen werden Bürger } \\
\text { von Interessengruppen manipuliert. }\end{array}$ & 70 & 66 \\
\hline $\begin{array}{l}\text { Minderheiten können bei der direkten Demokratie eher } \\
\text { eine Mehrheit bekommen als bei einer repräsentativen } \\
\text { Demokratie. }\end{array}$ & 63 & 63 \\
\hline $\begin{array}{l}\text { Eine Verlangsamung des politischen } \\
\text { Entscheidungsprozesses. }\end{array}$ & 67 & 62 \\
\hline $\begin{array}{l}\text { Die benötigten Unterschriften für Volksentscheide sind } \\
\text { zu schwierig zu erreichen. }\end{array}$ & 49 & 45 \\
\hline
\end{tabular}

Quelle: Eigene Darstellung; In \% der Anzahl der Personen, die der Befürchtung zustimmen.

In Tabelle 6 sind die Befürchtungen der Politiker und der Bevölkerung gegenüber der direkten Demokratie dargestellt. Bei beiden Akteursgruppen ist die Befürchtung stark ausgeprägt, dass direkte Demokratie den Interessengruppeneinfluss erleichtern würde: 81 Prozent der Politiker und 75 Prozent der Bürger erscheint eine zunehmende Macht von Interessengruppen, hervorgerufen durch die Einführung der direkten Demokratie, problematisch. Etwa 70 Prozent der Politiker und der Bürger befürchten sogar eine Manipulation durch die Interessengruppen bei direktdemokratischen Entscheidungen. In ähnlichem Maße sehen Politiker und Bürger den übermäßigen Einfluss von Minderheiten als relativ unproblematisch, die Verlangsamung politischer Prozesse als eher problematisch an. Die Verengung auf ein schlichtes Ja oder Nein in schwierigen politischen Fragen bereitet hingegen mit 81 Prozent vor allem den Politikern Unbehagen. Beide Akteursgruppen sehen deutlich, dass ohne Zustimmung politischer Mandatsträger keine Einführung der direkten Demokratie auf Bundesebene möglich 
ist, wobei das dahingehende Unbehagen bei den Bürgern überwiegt. Schließlich finden weniger als die Hälfte der Befragten die diskutierten Unterschriftenquoren problematisch.

\section{E. Ergebnisse der Regressionsanalyse}

Auf Basis dieser deskriptiven Analysen präsentieren die Tabellen 7 und 8 die Regressionsergebnisse für die Politiker und die Bevölkerung. Es werden vier Spezifikationen unterschieden, um die Effekte der bereits genannten Aufteilung Deutschlands in Ost und West, Süden und Norden/Mitte, konservativ und linksregierte Länder, kleine und große Länder zu analysieren. Gleichzeitig wird dadurch die Robustheit der Einflüsse der anderen unabhängigen Variablen unter diesen veränderten Bedingungen getestet.

\section{Soziodemographische Variablen}

Hinsichtlich der Bildung der Politiker lässt sich ein robuster signifikanter Effekt feststellen. Politiker, die mindestens Abitur haben, sind mit einer 14 bis $15 \%$ höheren Wahrscheinlichkeit negativer gegenüber der Einführung der direkten Demokratie eingestellt als Politiker ohne Abitur. Bei der Bevölkerung kann kein signifikanter Einfluss der Bildungsvariablen festgestellt werden. Ein robuster Einfluss des Alters auf die Einstellung zur direkten Demokratie kann ebenfalls nicht berichtet werden. Eher ist zu vermuten, dass eine breite Masse der unzufriedenen Bevölkerung die direkte Demokratie unterstützt, wobei keine Eingrenzungen auf bestimmte Altersgruppen gemacht werden können. ${ }^{33}$

Ein robuster positiver Effekt der Landes- und Bundesebene zeigt, dass Politiker, die auf Bundes- und Landesebene aktiv sind, sich im Vergleich zur Referenzgruppe der Kommunalebene mit einer 8 bis 9\% höheren Wahrscheinlichkeit für die direkte Demokratie auf Bundesebene aussprechen. Möglicherweise können sich Bundes- und Landtagsabgeordnete aufgrund einer besseren Kenntnis der Prozesse auf Bundesebene eine Einführung direktdemokratischer Mechanismen eher vorstellen als Kommunalpolitiker. Wie weitere nicht aufgeführte

33 Zur genaueren Betrachtung der Variable Alter wurde in einem aus Platzgründen nicht aufgeführten Modell die Variable Alter ${ }^{2}$ zusätzlich verwendet. Dabei wurde ebenfalls kein signifikanter Koeffizient festgestellt. Die Schätzergebnisse zusätzlicher im Text angesprochener Modelle stellen wir auf Anfrage bereit. 
Schätzergebnisse verdeutlichen, sprechen sich insbesondere Bundestagsabgeordnete am stärksten für direkte Demokratie aus. Ersetzt man die Variable zur politischen Ebene, um den Effekt der Bundesebene zu identifizieren, so lässt sich ein signifikant positiver, im Vergleich zur Landesebene bedeutsamerer Effekt der Bundesebene feststellen.

Tabelle 7: Probit-Modell zur Einstellung der Politiker zur direkten Demokratie

Wie bewerten Sie im Großen und Ganzen eine Einführung der direkten Demokratie in Deutschland auf Bundesebene?

\begin{tabular}{|c|c|c|c|c|c|c|c|c|}
\hline \multirow[b]{2}{*}{ Variable } & \multicolumn{2}{|c|}{ (1) } & \multicolumn{2}{|c|}{ (2) } & \multicolumn{2}{|c|}{ (3) } & \multicolumn{2}{|c|}{ (4) } \\
\hline & $\begin{array}{l}\text { Koeffizient/ } \\
\text { Std.-Fehler }\end{array}$ & $\begin{array}{l}\text { Marginaler } \\
\text { Effekt }\end{array}$ & $\begin{array}{l}\text { Koeffizient/ } \\
\text { Std.-Fehler }\end{array}$ & $\begin{array}{l}\text { Marginaler } \\
\text { Effekt }\end{array}$ & $\begin{array}{l}\text { Koeffizient/ } \\
\text { Std.-Fehler }\end{array}$ & $\begin{array}{c}\text { Marginaler } \\
\text { Effekt }\end{array}$ & $\begin{array}{l}\text { Koeffizient/ } \\
\text { Std.-Fehler }\end{array}$ & $\begin{array}{c}\text { Marginaler } \\
\text { Effekt }\end{array}$ \\
\hline \multicolumn{9}{|c|}{ Soziodemographische Variablen } \\
\hline Geschlecht & $\begin{array}{c}0,820 \\
(0,660)\end{array}$ & 0,053 & $\begin{array}{c}0,844 \\
(0,696)\end{array}$ & 0,055 & $\begin{array}{c}0,617 \\
(0,729)\end{array}$ & 0,040 & $\begin{array}{c}0,623 \\
(0,729)\end{array}$ & 0,040 \\
\hline Alter & $\begin{array}{l}-0,048 \\
(0,030)\end{array}$ & $-0,003$ & $\begin{array}{l}-0,043 \\
(0,031)\end{array}$ & $-0,003$ & $\begin{array}{l}-0,052^{*} \\
(0,030)\end{array}$ & $-0,003 *$ & $\begin{array}{l}-0,051^{*} \\
(0,030)\end{array}$ & $-0,003^{*}$ \\
\hline Bildung & $\begin{array}{c}-2,265^{* * *} \\
(0,870)\end{array}$ & $-0,147 * * *$ & $\begin{array}{c}-2,193 * * \\
(0,948)\end{array}$ & $-0,142 * *$ & $\begin{array}{c}-2,345^{* * * *} \\
(0,874)\end{array}$ & $-0,151 * * *$ & $\begin{array}{c}-2,335^{* * *} \\
(0,886)\end{array}$ & $-0,150 * * *$ \\
\hline $\begin{array}{l}\text { Landes- und } \\
\text { Bundesebene }\end{array}$ & $\begin{array}{l}1,388^{* *} \\
(0,592)\end{array}$ & $0,090 * *$ & $\begin{array}{l}1,298^{* *} \\
(0,585)\end{array}$ & $0,084 * *$ & $\begin{array}{l}1,322^{* *} \\
(0,602)\end{array}$ & $0,085 * *$ & $\begin{array}{l}1,330^{* *} \\
(0,602)\end{array}$ & $0,086 * *$ \\
\hline $\begin{array}{l}\text { Ostdeutsch- } \\
\text { land }\end{array}$ & $\begin{array}{c}0,485 \\
(1,201)\end{array}$ & 0,031 & & & & & & \\
\hline $\begin{array}{l}\text { Südliche } \\
\text { (Bundes)- } \\
\text { Länder }\end{array}$ & & & $\begin{array}{l}-0,729 \\
(0,521)\end{array}$ & $-0,047$ & & & & \\
\hline $\begin{array}{l}\text { Konservativ } \\
\text { regierte } \\
\text { Länder }\end{array}$ & & & & & $\begin{array}{c}-0,837 \\
(0,817)\end{array}$ & $-0,054$ & & \\
\hline $\begin{array}{l}\text { Große } \\
\text { Länder }\end{array}$ & & & & & & & $\begin{array}{l}-0,818 \\
(0,763)\end{array}$ & $-0,053$ \\
\hline \multicolumn{9}{|c|}{ Ideologische Ausrichtung } \\
\hline $\begin{array}{l}\text { Konservative } \\
\text { Parteien }\end{array}$ & $\begin{array}{l}-1,265^{*} \\
(0,663)\end{array}$ & $-0,082 *$ & $\begin{array}{l}-1,153^{*} \\
(0,699)\end{array}$ & $-0,075^{*}$ & $\begin{array}{l}-1,175^{*} \\
(0,714)\end{array}$ & $-0,076^{*}$ & $\begin{array}{l}-1,191^{*} \\
(0,697)\end{array}$ & $-0,077^{*}$ \\
\hline \multicolumn{9}{|c|}{ Blockadepotential } \\
\hline $\begin{array}{l}\text { Souveränität } \\
\text { der Politiker } \\
\text { wird einge- } \\
\text { schränkt } \\
\text { durch } \\
\text { direkte } \\
\text { Demokratie }\end{array}$ & $\begin{array}{c}-2,998 * * \\
(1,462)\end{array}$ & $-0,194 * *$ & $\begin{array}{c}-2,973 * * \\
(1,423)\end{array}$ & $-0,192 * *$ & $\begin{array}{c}-3,107 * * \\
(1,417)\end{array}$ & $-0,200 * *$ & $\begin{array}{c}-3,088 * * \\
(1,425)\end{array}$ & $-0,198 * *$ \\
\hline
\end{tabular}




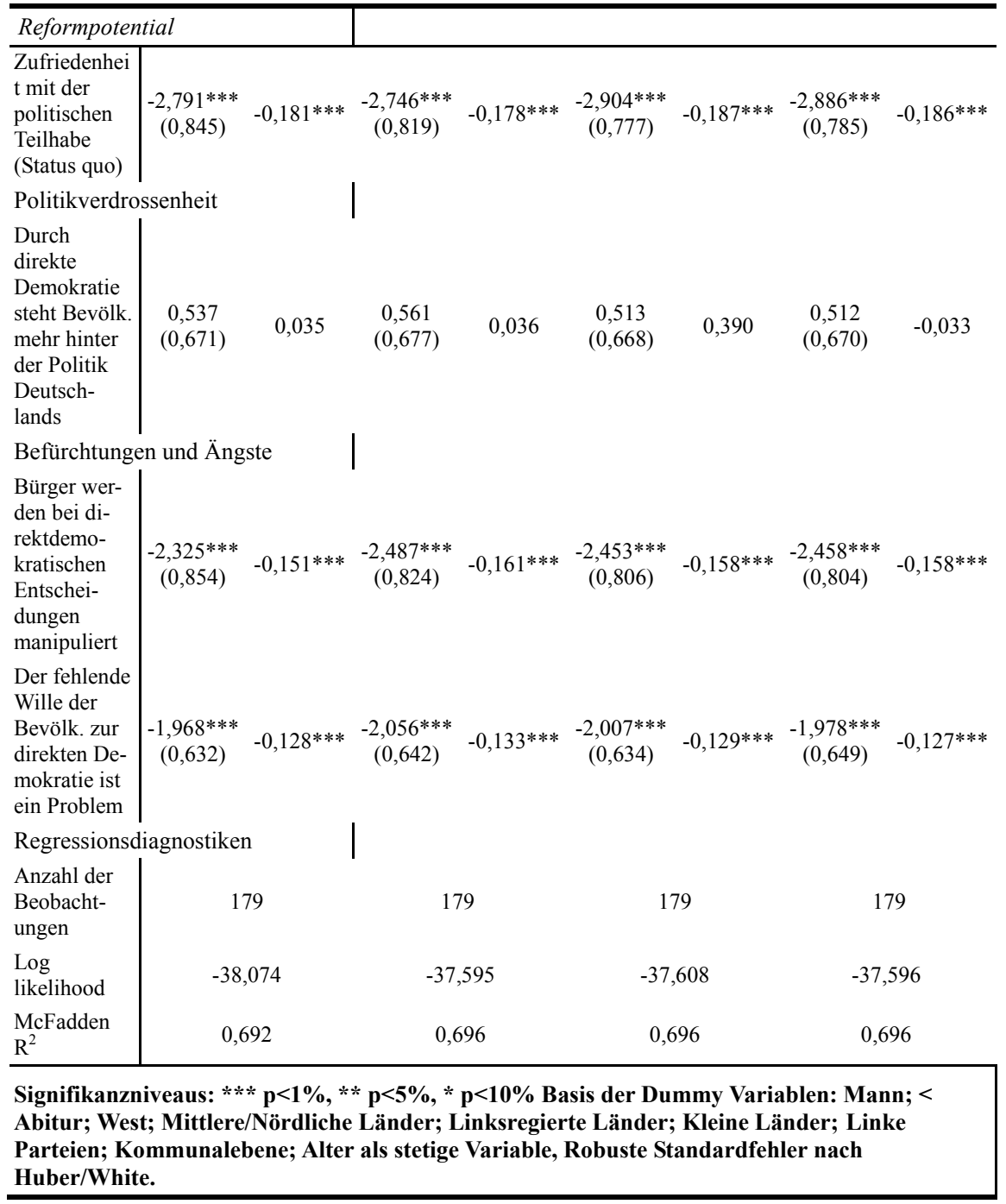


Tabelle 8: Probit-Modell zur Einstellung der Bevölkerung zur direkten Demokratie

Wie bewerten Sie im Großen und Ganzen eine Einführung der direkten Demokratie in Deutschland auf Bundesebene?

\begin{tabular}{|c|c|c|c|c|c|c|c|c|}
\hline \multirow[b]{2}{*}{ Variable } & \multicolumn{2}{|c|}{ (1) } & \multicolumn{2}{|c|}{ (2) } & \multicolumn{2}{|c|}{ (3) } & \multicolumn{2}{|c|}{ (4) } \\
\hline & \begin{tabular}{|l} 
Koeffizient/ \\
Std.-Fehler
\end{tabular} & $\begin{array}{c}\text { Marginaler } \\
\text { Effekt }\end{array}$ & $\begin{array}{l}\text { Koeffizient/ } \\
\text { Std.-Fehler }\end{array}$ & $\begin{array}{c}\text { Marginaler } \\
\text { Effekt }\end{array}$ & $\begin{array}{l}\text { Koeffizient/ } \\
\text { Std.-Fehler }\end{array}$ & $\begin{array}{c}\text { Marginaler } \\
\text { Effekt }\end{array}$ & $\begin{array}{l}\text { Koeffizient/ } \\
\text { Std.-Fehler }\end{array}$ & $\underset{\text { Effekt }}{\text { Marginaler }}$ \\
\hline \multicolumn{9}{|c|}{ Soziodemographische Variablen } \\
\hline Geschlecht & \begin{tabular}{|c}
0,665 \\
$(0,738)$
\end{tabular} & 0,057 & $\begin{array}{c}0,819 \\
(0,810)\end{array}$ & 0,067 & $\begin{array}{c}0,939 \\
(0,895)\end{array}$ & 0,076 & $\begin{array}{c}0,747 \\
(0,875)\end{array}$ & 0,061 \\
\hline Alter & $\begin{array}{l}-0,003 \\
(0,020)\end{array}$ & $-0,000$ & $\begin{array}{l}-0,008 \\
(0,022)\end{array}$ & $-0,001$ & $\begin{array}{l}-0,007 \\
(0,021)\end{array}$ & $-0,001$ & $\begin{array}{c}-0,004 \\
(0,021)\end{array}$ & $-0,000$ \\
\hline Bildung & $\begin{array}{l}-0,190 \\
(0,865)\end{array}$ & $-0,016$ & $\begin{array}{c}0,009 \\
(0,863)\end{array}$ & 0,001 & $\begin{array}{l}-0,309 \\
(0,845)\end{array}$ & $-0,025$ & $\begin{array}{l}-0,079 \\
(0,890)\end{array}$ & $-0,006$ \\
\hline $\begin{array}{l}\text { Ostdeutsch- } \\
\text { land }\end{array}$ & $\begin{array}{c}1,038 \\
(1,080)\end{array}$ & 0,089 & & & & & & \\
\hline $\begin{array}{l}\text { Südliche } \\
\text { Bundesländer }\end{array}$ & & & $\begin{array}{c}-1,852 \\
(1,261)\end{array}$ & $-0,151$ & & & & \\
\hline $\begin{array}{l}\text { Konservativ } \\
\text { regierte } \\
\text { Bundesländer }\end{array}$ & & & & & $\begin{array}{c}-1,599^{* *} \\
(0,759)\end{array}$ & $-0,130 * *$ & & \\
\hline $\begin{array}{l}\text { Große } \\
\text { Bundesländer }\end{array}$ & & & & & & & $\begin{array}{l}-1,588^{*} \\
(0,827)\end{array}$ & $-0,129 *$ \\
\hline \multicolumn{9}{|c|}{ Reformpotential } \\
\hline $\begin{array}{l}\text { Zufriedenheit } \\
\text { mit der } \\
\text { politischen } \\
\text { Teilhabe }\end{array}$ & $\begin{array}{l}-0,935 \\
(0,784)\end{array}$ & $-0,080$ & $\begin{array}{l}-1,376 \\
(0,982)\end{array}$ & $-0,112$ & $\begin{array}{l}-0,979 \\
(0,852)\end{array}$ & $-0,080$ & $\begin{array}{c}-1,032 \\
(0,855)\end{array}$ & $-0,084$ \\
\hline $\begin{array}{l}\text { Mehr } \\
\text { Teilhabe } \\
\text { verbinde ich } \\
\text { mit direkter } \\
\text { Demokratie }\end{array}$ & $\begin{array}{c}3,071 * * * \\
(0,870)\end{array}$ & $0,263 * * *$ & $\begin{array}{c}2,870 * * * \\
(0,803)\end{array}$ & $0,234 * * *$ & $\begin{array}{c}3,135 * * * \\
(1,013)\end{array}$ & $0,255^{* * *}$ & $\begin{array}{c}3,368 * * * \\
(0,929)\end{array}$ & $0,274 * * *$ \\
\hline \multicolumn{9}{|c|}{ Politikverdrossenheit } \\
\hline $\begin{array}{l}\text { Durch direkte } \\
\text { Demokratie } \\
\text { steht Bevölk. } \\
\text { mehr hinter } \\
\text { der Politik } \\
\text { Deutschlands }\end{array}$ & $\begin{array}{c}2,269 * * \\
(0,996)\end{array}$ & $0,194 * *$ & $\begin{array}{c}2,374 * * \\
(1,055)\end{array}$ & $0,194 * *$ & $\begin{array}{c}2,189^{* *} \\
(1,035)\end{array}$ & $0,178 * *$ & $\begin{array}{l}1,141 * * \\
(1,050)\end{array}$ & $0,174^{* *}$ \\
\hline $\begin{array}{l}\text { Weniger } \\
\text { Poli- } \\
\text { tikverdruss } \\
\text { bei Einfüh- } \\
\text { rung der dir. } \\
\text { Demokratie }\end{array}$ & $\begin{array}{c}2,341 * * * \\
(0,791)\end{array}$ & $0,201 * * *$ & $\begin{array}{c}2,425^{* *} \\
(0,968)\end{array}$ & $0,198^{* *}$ & $\begin{array}{c}2,483 * * * \\
(0,954)\end{array}$ & $0,202 * * *$ & $\begin{array}{c}2,439 * * * \\
(0,878)\end{array}$ & $0,198 * * *$ \\
\hline
\end{tabular}




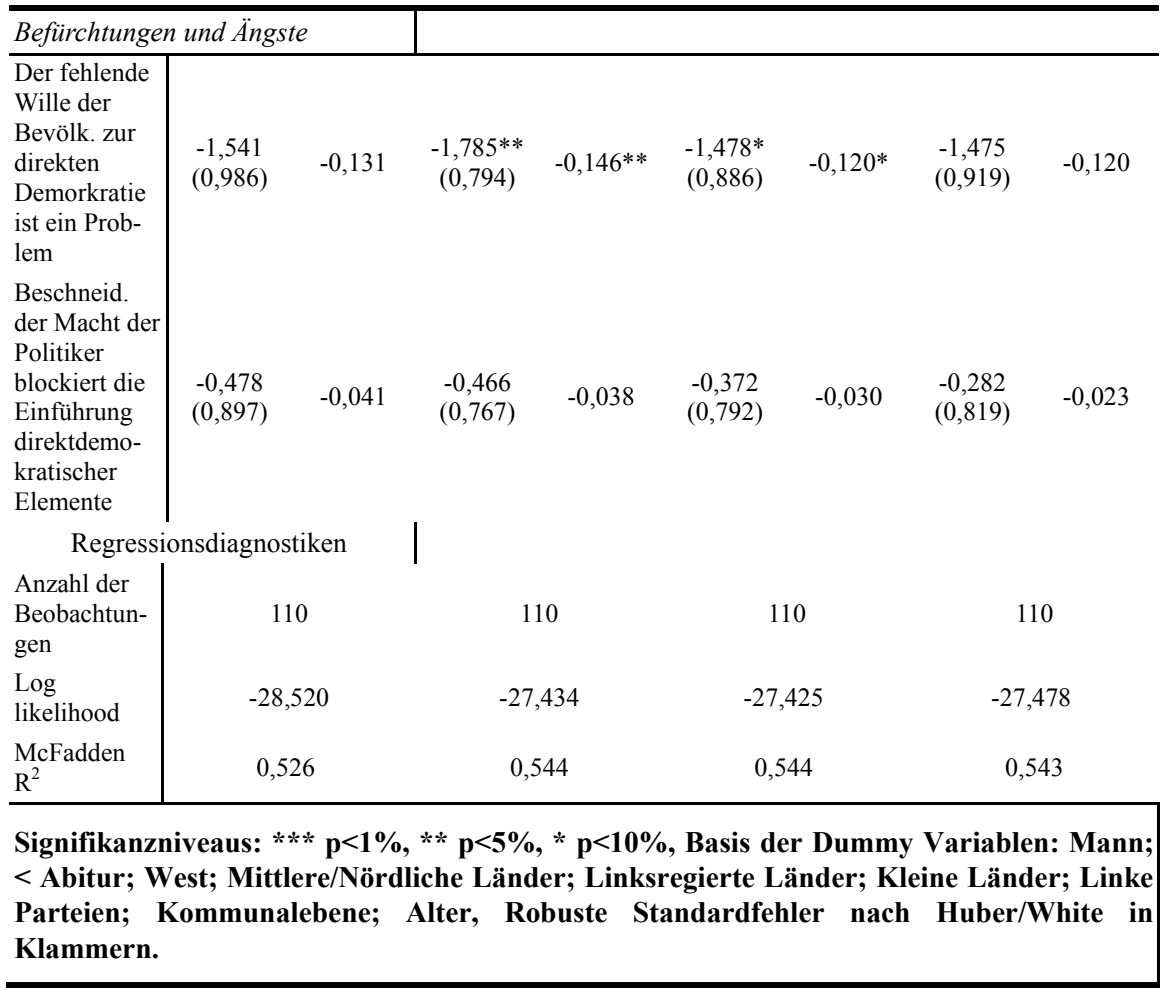

Die Schätzergebnisse für die Einstellungen der Bürger lassen deutliche Unterschiede zu den Politikern erkennen. Dass die ostdeutsche Bevölkerung positiver gegenüber der direkten Demokratie eingestellt ist, lässt sich gemäß unseren Schätzergebnissen zwar nicht stützen. Ein signifikant negativer marginaler Effekt auf die Einstellung zur direkten Demokratie der Bevölkerung kann jedoch für konservativ regierte Länder sowie für große Bundesländer festgestellt werden. Die Bürger großer Bundesländer scheinen Schwierigkeiten damit zu haben, sich direkte Demokratie bundesweit vorzustellen.

II. Ideologische Präferenzen und Blockadepotential der Politiker

Ein robuster, aber geringer negativer marginaler Effekt von -0,08 zeigt sich bei den Politikern aus ,konservativen Parteien“. Dies bestätigt den erwarteten nega- 
tiven Einfluss von CDU und CSU. Wie vorausgesagt, stellt sich der Souveränitätsverlust bei den Politikern als robuster negativer Einfluss auf die Einstellung zur direkten Demokratie heraus. Dabei nimmt der marginale Effekt einen Wert von $-0,2$ an und stellt somit den quantitativ stärksten Einflussfaktor dar. Politiker, die sich durch eine Einführung direktdemokratischer Elemente auf Bundesebene in ihrer Souveränität eingeschränkt fühlen, stimmen mit einer um 20 Prozent geringeren Wahrscheinlichkeit für die Einführung der direkten Demokratie auf Bundesebene.

\section{Reformpotential}

Dass das Reformpotential oder genauer die Zufriedenheit mit dem Status quo bei den Politikern eine sehr große Rolle spielt, zeigt sowohl die Höhe der marginalen Effekte als auch ihre Signifikanz. Ein Politiker, der zufrieden mit der politischen Teilhabe ist, spricht sich mit einer um 18 Prozent geringeren Wahrscheinlichkeit für direkte Volksrechte auf Bundesebene aus als ein Politiker, der nicht zufrieden mit dem Status quo ist. Solange die Politiker keinen Grund haben, mit dem Status Quo unzufrieden zu sein, haben Reformen, die den Status quo verändern, eine geringe Chance. Dies ist auch angesichts ihrer Möglichkeiten zur politischen Teilhabe nicht verwunderlich.

Ein solch starker und signifikanter Effekt kann für die Einstellung der Bevölkerung zur direkten Demokratie nicht festgestellt werden. Den größten Einfluss darauf nimmt vielmehr die zweite Variable des Reformpotentials. So hat die Überzeugung der Bevölkerung, dass durch die direkte Demokratie eine stärkere Teilhabe bei politischen Entscheidungen erreicht werden kann, einen robusten positiven marginalen Effekt von 0,23 bis 0,27. Die Kenntnis über die Auswirkungen der direkten Demokratie verstärkt auch die Einstellungen zur direkten Demokratie und unterstützt dadurch eine Tendenz weg vom Status quo. Betrachtet man beide Variablen zur Abbildung des Reformpotentials für die Bevölkerung gemeinsam, so kann insgesamt zwar kein Reformpotential aufgrund der Unzufriedenheit unter der Bevölkerung festgestellt werden, dafür jedoch umso stärker durch die Möglichkeit der politischen Teilhabe in der direkten Demokratie. Es ist also eher der Wunsch nach aktiver Mitgestaltung als die Frustration über den gegenwärtigen Zustand der deutschen Politik, der die Bürger zu einer Befürwortung der Einführung direkter Demokratie auf Bundesebene veranlasst. 
Diese Unzufriedenheit mit dem Status quo ist von einer generellen Politikverdrossenheit zu unterscheiden. Bei der Bevölkerung lassen sich deutliche signifikant positive Effekte von bis zu 0,2 bei beiden Variablen zur Erfassung der Politikverdrossenheit feststellen. Insbesondere befürworten diejenigen Bürger eine Reform, die bei Einführung der direkten Demokratie erwarten, dass die Bevölkerung stärker hinter der Politik stehen würde oder bereit wäre, sich intensiver mit politischen Themen auseinander zu setzen. Dies stützt die Argumente von Russell et al. ${ }^{34}$, dass politikverdrossene Menschen sich nach mehr direkter Demokratie sehnen und ein gewisses Protestpotential gegen das rein repräsentative Modell entwickeln. Man könnte vermuten, dass ein Rückgang der Politikverdrossenheit durch Einführung der direkten Demokratie die Politiker zu deren Unterstützung bewegt. Ein solcher Effekt kann jedoch nicht festgestellt werden. Dies könnte unter anderem daran liegen, dass die Politiker nicht wirklich an eine Verdrängung der Politikverdrossenheit glauben.

\section{Befürchtungen und Ängste}

Wie erwartet, bestimmt die Befürchtung, dass die Bevölkerung durch Interessengruppen in der direkten Demokratie leichter manipuliert werden kann, in hohem Maße die Einstellungen der Politiker: Diejenigen, die einen größeren Einfluss der Interessengruppen bei Volksabstimmungen befürchten, sprechen sich mit einer 15 bis $16 \%$ geringeren Wahrscheinlichkeit für die Einführung direkter Volksrechte auf Bundesebene aus. Zudem sehen Politiker auch einen fehlenden Willen auf Seiten der Bevölkerung zur Einführung der direkten Demokratie als einen Grund an, sich gegen direkte Demokratie auszusprechen. Auf Seiten der Bevölkerung zeigt sich jedoch kein robuster signifikanter Effekt solcher Ängste. Auch wirken sich Befürchtungen einer Blockadehaltung der Politiker nicht auf die Zustimmung der Bürger zur direkten Demokratie aus. 
Während sich die individuellen sozio-demographischen Charakteristika sowohl bei den Politikern als auch bei der Bevölkerung nur in geringem Maße auf die Einstellungen zur direkten Demokratie auswirken, können in den restlichen Kategorien robuste und signifikante Effekte festgestellt werden. Hinsichtlich ideologischer Präferenzen ist deutlich zu erkennen, dass CDU und CSU die direkte Demokratie ablehnen. Ein gewisses Reformpotential ist durch den Wunsch der Bevölkerung nach mehr Teilhabe zu erkennen, während bei den Politikern eher die Zufriedenheit mit dem Status quo dominiert. Dies spiegelt sich besonders in einem Blockadepotential aufgrund des befürchteten Souveränitätsverlusts wider, wie auch in den deutlich negativen Einflüssen der Befürchtungen zur direkten Demokratie. Weiterhin zeigt sich, dass die Aussicht auf eine geringere Politikverdrossenheit keinen signifikanten Einfluss auf die Einstellungen der Politiker hat, während sich jedoch die Vorstellung von mehr politischer Teilhabe positiv auf die Einstellung der Bevölkerung zur direkten Demokratie auswirkt.

\section{F. Fazit und Ausblick}

In diesem Beitrag haben wir die Einstellungen der Bürger und der Politiker zur Einführung direkter Volksrechte auf Bundesebene auf Basis einer Befragung unter diesen Akteursgruppen untersucht. Es lässt sich feststellen, dass die Bevölkerung die direkte Demokratie für die Bundesebene mit einer Zustimmung von 75 Prozent befürwortet. Diese Zustimmung hängt zum einen von der Hoffnung auf mehr Mitbestimmung und zum anderen vom Ausmaß an Politikverdrossenheit ab. Zudem sprechen sich Bürger in konservativ regierten oder in großen Bundesländern in signifikant geringerem Maße für die direkte Demokratie aus.

Hingegen lehnen 56 Prozent der befragten Politiker eine Einführung direkter Demokratie auf Bundesebene ab. Besonders stark ist diese Ablehnung unter den Mitgliedern konservativer Parteien ausgeprägt. Diejenigen Repräsentanten, die einen Souveränitätsverlust oder eine Manipulation der Bürger durch Interessengruppen bei Volksabstimmungen befürchten, sprechen sich gegen die Einführung direkter Volksrechte aus. Vor allem Politiker mit höheren Bildungsabschlüssen sind gegen die direkte Demokratie eingestellt. Vermutlich befürchten sie, dass politische Entscheidungen mit einem zu geringen Informationsstand der Bevölkerung in der direkten Demokratie entschieden werden.

Im Vergleich der Einstellungen der beiden Akteursgruppen und der Gründe für ihre Einstellungen wird deutlich, dass dem Wunsch der Bevölkerung nach 
mehr direkter Demokratie auf Bundesebene eine ganze Reihe von Befürchtungen auf Seiten der Politiker entgegensteht. Angesichts der internationalen Evidenz zum Einfluss von Interessengruppen in der direkten und repräsentativen Demokratie, zur Informationsproblematik oder zu den ökonomischen Effekten ${ }^{35}$ lassen sich möglicherweise manche dieser Zweifel beseitigen. Letztlich bleibt als bedeutsames Hindernis für die Einführung direkter Demokratie auf Bundesebene, dass Politiker in gewissem Maße ihre politische Entscheidungssouveränität mit den Bürgern teilen müssen. Dieses Hindernis lässt sich durch keine Argumente und durch keine wissenschaftliche Evidenz beseitigen: es ist vielmehr inhärenter Bestandteil der verfassungspolitischen Empfehlung zur Einführung direkter Volksrechte. Jedenfalls lässt sich angesichts dieser Befunde nicht behaupten, die Bürger wollten keine direkte Demokratie. Vermutlich ist die Annahme von Calliess $^{36}$ zutreffend, dass die Deutschen ihr Grundgesetz nicht aufgeben möchten. Dies wäre jedoch keine hinreichende Begründung gegen seine Veränderung.

35 Gerber, The Populist Paradox (Fn. 16); Kirchgässner/Feld/Savioz, Direkte Demokratie (Fn. 11); Matsusaka, For the Many (Fn. 16).

36 Calliess, Ringen (Fn. 5). 